\title{
Correction for Foreshortening in Optical Odontometry
}

\author{
STEPHEN M. BAILEY, STANLEY M. GARN, and ROBERT L. WAINRIGHT
}

Center for Human Growth and Development, The University of Michigan, Ann Arbor, Michigan 48109 U. S. A.

\section{J Dent Res 58(1):539, January 1979}

Increasing use of optical odontometric techniques (cf. Biggerstaff, Angle Orthod., 40:28-36, 1970; Amer. J. Phys. Anthrop., 31:163, 1969; Corruccini, J. Dent. Res., 56:699, 1977; Van der Linden et al., $J$. Dent. Res., 51:1100, 1972) raises the question of to what degree measurements oriented to an optically foreshortened plane are comparable to those taken manually at the crown surface.

We have investigated this problem using University of Michigan School Growth Study dental cast data, collected with the OPTOCOM (Van der Linden et al., ibid.). Measurements of 2397 molar and premolar mesial buccolingual cusp tip to cusp tip diameters, from 204 persons, were used. Because the OPTOCOM gives triaxial ( $X, Y$ and $Z$ ) coordinates, it was possible geometrically to reconstruct the differences between the foreshortened OPTOCOM measurements and conventional crown surface measurements of the same casts. The corrected diameter represents the hypotenuse of a right triangle completed by the mesial buccolingual cusp height differences and the horizontal (optical) plane. To validate use of the geometrically reconstructed surface planes as equivalent to the actual surface plane, measured with calipers, one of us (S. B.) measured a subsample of casts using both methods. Agreement between geometrically and manually measured surface planes was within one percent.

When the optical plane diameters were compared to those from the geometrically reconstructed surface planes, for 2397 teeth, differences

Received for publication April 14, 1978.

Accepted for publication May 18, 1978.

from $1 / 2$ to over 17 percent were found, as shown in the Table. These systematic differences, for 16 premolars and molars in both sexes, are all highly significant statistically $(p<.0001)$. While leftright effects were negligible, as might be expected, mandibular differences were considerably greater than those in the maxilla.

While these data do not allow differentiation of these measurement differences into components caused by tooth angulation, and by cusp height variations, the summed buccolingual angulation from the horizontal (e.g., degrees of foreshortening) was calculated, and is indeed substantial, reaching 34 degrees for the mandibular P1. Only 11.5 degrees of total angulation will lead to a 2 percent difference between the optical and surface values.

These differences, created by foreshortening, could lead to an incorrect assertion of statistical differences when comparing odontometric samples taken manually and optically. Where investigators using optical plane surface measurements wish to make such a comparison, or to determine the total angulation of a tooth, triaxial measurements can provide suitable corrections. However, if the height measurement ( $Y$ axis) is unavailable, we offer the following formula, requiring only a protractor and access to trigonometric function tables or a suitable calculator, to convert optical plane diameters:

Surface diameter $=$ Optical diameter Cosine of summed angulation

\section{Acknowledgment.}

This study was supported in part by Grant DE-03610 from the National Institutes of Health. TABLE

COMPARISON OF OPTICAL AND SURFACE PLANE B-L CUSP TO CUSP DIAMETERS

\begin{tabular}{|c|c|c|c|c|c|c|c|c|c|}
\hline \multirow{2}{*}{\multicolumn{2}{|c|}{$\frac{\text { Tooth }}{\text { Maxilla }}$}} & \multicolumn{4}{|c|}{ Males } & \multicolumn{4}{|c|}{ Females } \\
\hline & & \multirow{2}{*}{ N } & \multirow{2}{*}{$\frac{\begin{array}{c}\text { optical plane } \\
\text { diameter }(\mathrm{mm})\end{array}}{6.33}$} & \multirow{2}{*}{$\underbrace{\begin{array}{c}\text { surface plane } \\
\text { diameter }(\mathrm{mm})\end{array}}_{6.45}$} & \multirow{2}{*}{$\frac{\% \text { difference }}{1}$} & \multirow{2}{*}{$\frac{\mathrm{N}}{53}$} & \multirow{2}{*}{$\begin{array}{c}\begin{array}{c}\text { optical plane } \\
\text { diameter }(\mathrm{mm})\end{array} \\
6.20\end{array}$} & \multirow{2}{*}{$\begin{array}{c}\begin{array}{c}\text { surface plane } \\
\text { diameter }(\mathrm{mm})\end{array} \\
6.36\end{array}$} & \multirow{2}{*}{$\frac{\% \text { difference }}{2.6}$} \\
\hline $\mathbf{R}$ & $\mathrm{M} 2$ & & & & & & & & \\
\hline $\mathbf{R}$ & MI & 109 & 6.21 & 6.30 & 1.5 & 99 & 6.04 & 6.16 & 2.0 \\
\hline$R$ & $\mathrm{P} 2$ & 65 & 5.46 & 5.49 & 0.5 & 50 & 5.40 & 5.43 & 0.6 \\
\hline $\mathrm{R}$ & P1 & 78 & 5.34 & 5.43 & 1.7 & 62 & 5.36 & 5.42 & 1.2 \\
\hline $\mathrm{L}$ & P1 & 79 & 5.36 & 5.45 & 1.7 & 59 & 5.34 & 5.40 & 1.1 \\
\hline $\mathrm{L}$ & P2 & 67 & 5.54 & 5.57 & 0.5 & 50 & 5.44 & 5.47 & 0.5 \\
\hline $\mathrm{L}$ & $\mathrm{MI}$ & 109 & 6.18 & 6.29 & 1.8 & 99 & 6.06 & 6.19 & 2.2 \\
\hline $\mathrm{L}$ & $\mathrm{M} 2$ & 73 & 6.23 & 6.37 & 2.2 & 54 & 6.05 & 6.23 & 2.9 \\
\hline \multicolumn{10}{|c|}{ Mandible } \\
\hline $\mathrm{R}$ & $\mathrm{M} 2$ & 82 & 4.70 & 4.99 & 5.9 & 57 & 4.61 & 4.88 & 5.6 \\
\hline $\mathrm{R}$ & M1 & 109 & 5.08 & 5.16 & 1.6 & 99 & 4.94 & 5.03 & 1.8 \\
\hline $\mathrm{R}$ & P2 & 66 & 4.26 & 4.52 & 5.8 & 47 & 4.36 & 4.56 & 4.4 \\
\hline $\mathrm{R}$ & P1 & 78 & 3.58 & 4.33 & 17.4 & 68 & 3.59 & 4.25 & 15.6 \\
\hline $\mathbf{L}$ & $\mathrm{P} 1$ & 80 & 3.62 & 4.39 & 17.5 & 66 & 3.75 & 4.37 & 14.2 \\
\hline $\mathrm{L}$ & $\mathrm{P} 2$ & 66 & 4.22 & 4.49 & 6.1 & 53 & 4.32 & 4.51 & 4.2 \\
\hline $\mathbf{L}$ & M1 & 109 & 5.06 & S..? & 1.6 & 99 & 4.97 & 5.07 & 2.0 \\
\hline $\mathrm{L}$ & M2 & 79 & 4.64 & 4.98 & 6.8 & 59 & 4.67 & 4.96 & 5.8 \\
\hline
\end{tabular}

\footnotetext{
1 All differences significant by matched pair t-test, $p<.0001$.
} 